\title{
Skinfold Thickness as an Indication of Maturity of the Newborn
}

\author{
VALERIE FARR \\ From the Department of Child Health, University of Aberdeen
}

The abundant subcutaneous fat of the mature newborn infant is laid down mainly in the last two months of intrauterine life (Parmelee 1959), and is characteristically lacking in the premature infant. Measurement of skinfold thickness might therefore be of value as an indicator of the maturity of a newborn infant. Few observations of neonatal skinfold thickness have been recorded. Vincent and Hugon (1962) limited their study to African infants weighing $2,500 \mathrm{~g}$. or less, and Singh and Venkatachalam (1962) measured Indian infants whose mothers were of low socioeconomic status. None of these authors related their findings to the duration of pregnancy. Gampel (1965) related skinfold thickness to the length of gestation, but his series was derived from births selected on medical and social grounds. The present study was undertaken to measure skinfold thickness using the calipers described by Verel and Kesterven (1960) and to relate the results to birth weight and gestational age; the work formed part of a larger study of the physical characteristics of the newborn infant.

\section{Procedure}

Skinfold thickness was measured in 300 infants of known gestational age. The length of pregnancy was considered to be satisfactorily established if the mother was sure of her dates, her last menstrual period was normal both in duration and amount, with no bleeding within a month of the date given, and she had a regular cycle of $28 \pm 2$ days. If there was any reason to doubt the reliability of the evidence, or if the condition of the baby did not permit detailed examination, the case was excluded from the series. Measurements were made on all infants fulfilling the criteria, who were born in Aberdeen Maternity Hospital during a period of 5 months from March 13, 1964, and during a further period of 3 months from January 11, 1965 . Nine infants weighing less than $2,268 \mathrm{~g}$. $(5 \mathrm{lb}$.) and born outside the above dates were also included in order to increase the numbers in this weight group. All measurements were made within 48 hours of birth, as Vincent and Hugon (1962)

Received August 23, 1965. found that skinfold thickness diminishes by several tenths of a millimetre with each day of age.

Five sites were chosen (a sixth, over the buttock, was soon abandoned, because it proved difficult to pick up a suitable skinfold, and the calipers tended to slip, causing discomfort). (1) The lower angle of the scapula, picking up a natural skinfold. (2) The posterior border of the upper arm, midway between the tip of the shoulder and the elbow. (3) The lowest rib margin, in the nipple line. (4) The anterior abdominal wall, just above the umbilicus. (5) The anterior aspect of the thigh, just above the knee.

The scapula and arm measurements were made with the elbow flexed to 90 degrees and the limb held against the trunk. Thigh measurements were made with the hip and the knee flexed to 90 degrees. All measurements were made on the left side, and each recorded measurement is the mean of three estimations.

The series comprised 162 males and 138 females. The birth weights ranged from $1,200 \mathrm{~g}$. $(2 \mathrm{lb} .10 \mathrm{oz}$.) to $5,190 \mathrm{~g}$. (10 lb. $7 \mathrm{oz}$.) with a mean value of $3,120 \mathrm{~g}$. (6 lb. $14 \mathrm{oz}$.) and a standard deviation of $624 \mathrm{~g}$. (1 lb. $6 \mathrm{oz}$.). The median weight was $3,175 \mathrm{~g}$. $(7 \mathrm{lb}$.) and the weight distribution was only slightly skewed (25th percentile $6 \mathrm{lb} .1 \mathrm{oz}$., and 75th percentile $7 \mathrm{lb} .15 \mathrm{oz}$.). The gestational ages ranged from 29 weeks 5 days, to 44 weeks, with a mean of 39 weeks and a standard deviation of $2 \cdot 2$ weeks. There were 11 sets of twins in the series ( 14 males and 8 females); their mean weight was $2,500 \mathrm{~g}$. $\left(5 \frac{1}{2} \mathrm{lb}\right.$.) and mean length of gestation 37 weeks.

\section{Results}

Table I shows the distribution of cases by birth weight, length of gestation, and sex, and Table II shows the mean values for skinfold thickness (in millimetres) at each site, the average for all five sites, and the standard deviation for all cases.

The mean value for the average of all five sites, taking males and females together, was $4.54 \mathrm{~mm}$. and the approximate median was $4.48 \mathrm{~mm}$. The 25 th percentile was $3.65 \mathrm{~mm}$. and the 75 th percentile was $5.37 \mathrm{~mm}$. As the distribution is only slightly skewed, straight measurements have been used rather than the log of the measurements. 
TABLE IA

Distribution of Cases by Length of Gestation, and Sex (number of twins in each group in parentheses)

\begin{tabular}{c|c|c|c}
\hline $\begin{array}{c}\text { Length of } \\
\text { Gestation (wk.) }\end{array}$ & Males & Females & Total \\
\hline$<32$ & 1 & 1 & 2 \\
$32-$ & 1 & 0 & 1 \\
$33-$ & 3 & 2 & 5 \\
$34-$ & $6(3)$ & $5(3)$ & $11(6)$ \\
$35-$ & 9 & 3 & 12 \\
$36-$ & $9(2)$ & 2 & $11(2)$ \\
$37-$ & $17(7)$ & $14(3)$ & $31(10)$ \\
$38-$ & 21 & 26 & 47 \\
$39-$ & $36(1)$ & $39(1)$ & $75(2)$ \\
$40-$ & $34(1)$ & $25(1)$ & $59(2)$ \\
$41-$ & 19 & 15 & 34 \\
42 and over & 6 & 6 & 12 \\
\hline & $162(14)$ & $138(8)$ & $300(22)$ \\
\hline
\end{tabular}

TABLE IB

Distribution of Cases by Birth Weight and Sex (number of twins is given in parentheses)

\begin{tabular}{|c|c|c|c|}
\hline Birth Weight & Males & Females & Total \\
\hline \multirow[t]{3}{*}{$\begin{array}{l}<4 \text { lb. }(1,814 \mathrm{~g} .) \\
4 \mathrm{lb} .(1,814 \mathrm{~g} .)- \\
5 \mathrm{lb} .(2,268 \mathrm{~g} .)- \\
6 \mathrm{lb} .(2,722 \mathrm{~g} .)- \\
7 \mathrm{lb} .(3,175 \mathrm{~g} .)- \\
8 \mathrm{lb} .(3,629 \mathrm{~g} .)- \\
9 \mathrm{lb}(4,083 \mathrm{~g} .)- \\
10 \mathrm{lb} \text {. }(4,540 \mathrm{~g} .) \\
\quad \text { and over }\end{array}$} & $\begin{array}{c}3 \\
11(4) \\
21(6) \\
41(4) \\
42 \\
31 \\
11\end{array}$ & $\begin{array}{r}4(1) \\
14(3) \\
18(2) \\
37(1) \\
38(1) \\
19 \\
7\end{array}$ & $\begin{array}{r}7(1) \\
25(7) \\
39(8) \\
78(5) \\
80(1) \\
50 \\
18\end{array}$ \\
\hline & 2 & 1 & 3 \\
\hline & $162(14)$ & $138(8)$ & $300(22)$ \\
\hline
\end{tabular}

TABLE II

Mean Values for Skinfold Thickness (mm.) at each Site, and the Average for all 5 Sites

\begin{tabular}{|c|c|c|c|c|}
\hline Site & Males & Females & All Cases & S.D. \\
\hline $\begin{array}{lll}\text { Scapula } & \ldots & \ldots \\
\text { Arm } & \ldots & \ldots \\
\text { Chest } & . & \ldots \\
\text { Abdomen } & & \ldots \\
\text { Thigh } & \text { The } & \ldots \\
\text { Average of five sites }\end{array}$ & $\begin{array}{l}4 \cdot 70 \\
4 \cdot 48 \\
3 \cdot 49 \\
3 \cdot 64 \\
5 \cdot 66 \\
4 \cdot 40\end{array}$ & $\begin{array}{l}5 \cdot 03 \\
4 \cdot 84 \\
3 \cdot 61 \\
3 \cdot 94 \\
6 \cdot 60 \\
4 \cdot 80\end{array}$ & $\begin{array}{l}4 \cdot 84 \\
4 \cdot 65 \\
3 \cdot 56 \\
3 \cdot 78 \\
6 \cdot 09 \\
4 \cdot 54\end{array}$ & $\begin{array}{l}1 \cdot 53 \\
1 \cdot 75 \\
0 \cdot 95 \\
1 \cdot 10 \\
1 \cdot 85 \\
1 \cdot 25\end{array}$ \\
\hline
\end{tabular}

Skinfold thickness is greater in the females than in the males at all sites. The highest reading is found over the anterior thigh $(5.66 \mathrm{~mm}$. for males, and $6.60 \mathrm{~mm}$. for females) and the lowest readings over the anterior chest wall and abdominal wall $(3.56 \mathrm{~mm}$. and $3.78 \mathrm{~mm}$., respectively). There is a wide scatter about the mean at all five sites.

When skinfold thickness is considered in relation to body weight (Table III and Fig. 1), there is an increase in mean values with increasing weight. This is most evident over the thigh, the increase being approximately $0.9 \mathrm{~mm}$. for every $1 \mathrm{lb}$. in weight and least over the chest wall $(0.3 \mathrm{~mm}$. per lb.). Taking the average for all five sites, the increase is $0.56 \mathrm{~mm}$. per lb. In females weighing over $5 \mathrm{lb}$. $(2,268 \mathrm{~g}$.), mean values are higher than in males at all sites, but under this weight the difference is reversed for measurements over the chest and abdominal wall. Given birth weight and length of gestation, the difference in skinfold thickness

TABLE III

Mean Values for Skinfold Thickness at 5 Sites, by Weight and Sex

\begin{tabular}{|c|c|c|c|c|c|c|c|c|c|}
\hline \multicolumn{2}{|c|}{ Birth Weight } & \multirow{2}{*}{ Sex } & \multirow{2}{*}{ No. } & \multirow{2}{*}{$\begin{array}{c}\text { Scapula } \\
\text { (mm.) }\end{array}$} & \multirow{2}{*}{$\underset{(\mathrm{mm} .)}{\operatorname{Arm}}$} & \multirow{2}{*}{$\begin{array}{l}\text { Chest } \\
\text { (mm.) }\end{array}$} & \multirow{2}{*}{$\begin{array}{l}\text { Abdomen } \\
\text { (mm.) }\end{array}$} & \multirow{2}{*}{$\begin{array}{l}\text { Thigh } \\
\text { (mm.) }\end{array}$} & \multirow{2}{*}{$\begin{array}{c}\text { Average } \\
\text { (mm.) }\end{array}$} \\
\hline lb. & g. & & & & & & & & \\
\hline \multirow[t]{2}{*}{$<5$} & \multirow[t]{2}{*}{$<2,268$} & $\underset{\mathrm{F}}{\mathrm{M}}$ & $\begin{array}{l}14 \\
18 \\
\end{array}$ & $\begin{array}{l}3 \cdot 44 \\
3 \cdot 83 \\
\end{array}$ & $\begin{array}{l}3 \cdot 38 \\
3 \cdot 62\end{array}$ & $\begin{array}{l}2 \cdot 98 \\
2 \cdot 84\end{array}$ & $\begin{array}{l}3 \cdot 06 \\
2 \cdot 73\end{array}$ & $\begin{array}{l}3 \cdot 73 \\
4 \cdot 06\end{array}$ & $\begin{array}{l}3 \cdot 32 \\
3 \cdot 43\end{array}$ \\
\hline & & $\mathbf{M}$ and $\mathbf{F}$ & 32 & $3 \cdot 66$ & $3 \cdot 52$ & $2 \cdot 90$ & $2 \cdot 87$ & $3 \cdot 91$ & $3 \cdot 38$ \\
\hline \multirow[t]{2}{*}{$5-$} & \multirow[t]{2}{*}{2,268} & $\underset{\mathbf{F}}{\mathbf{M}}$ & $\begin{array}{l}21 \\
18 \\
\end{array}$ & $\begin{array}{l}4 \cdot 11 \\
3 \cdot 93 \\
\end{array}$ & $\begin{array}{l}3 \cdot 83 \\
4 \cdot 01 \\
\end{array}$ & $\begin{array}{l}3 \cdot 08 \\
3 \cdot 13 \\
\end{array}$ & $\begin{array}{l}3 \cdot 23 \\
3 \cdot 37 \\
\end{array}$ & $\begin{array}{l}4 \cdot 83 \\
5 \cdot 16 \\
\end{array}$ & $\begin{array}{l}3 \cdot 77 \\
4 \cdot 14 \\
\end{array}$ \\
\hline & & $M$ and $F$ & 39 & $4 \cdot 03$ & $3 \cdot 92$ & $3 \cdot 10$ & $3 \cdot 30$ & $4 \cdot 98$ & $3 \cdot 94$ \\
\hline \multirow[t]{2}{*}{$6-$} & \multirow[t]{2}{*}{$2,722-$} & $\underset{\mathrm{F}}{\mathrm{M}}$ & $\begin{array}{l}41 \\
37 \\
\end{array}$ & $\begin{array}{l}4 \cdot 26 \\
4 \cdot 79\end{array}$ & $\begin{array}{l}4 \cdot 14 \\
4 \cdot 56 \\
\end{array}$ & $\begin{array}{l}3 \cdot 23 \\
3 \cdot 42 \\
\end{array}$ & $\begin{array}{l}3 \cdot 38 \\
3 \cdot 67 \\
\end{array}$ & $\begin{array}{l}5 \cdot 02 \\
5 \cdot 96 \\
\end{array}$ & $\begin{array}{l}4 \cdot 01 \\
4 \cdot 37\end{array}$ \\
\hline & & $M$ and $F$ & 78 & $4 \cdot 49$ & $4 \cdot 34$ & $3 \cdot 32$ & $3 \cdot 52$ & $5 \cdot 47$ & $4 \cdot 18$ \\
\hline \multirow[t]{2}{*}{$7-$} & \multirow[t]{2}{*}{$3,175-$} & $\underset{\mathbf{F}}{\mathrm{M}}$ & $\begin{array}{l}42 \\
38 \\
\end{array}$ & $\begin{array}{l}4 \cdot 81 \\
5 \cdot 46 \\
\end{array}$ & $\begin{array}{l}4 \cdot 71 \\
5 \cdot 26 \\
\end{array}$ & $\begin{array}{l}3 \cdot 59 \\
3 \cdot 66 \\
\end{array}$ & $\begin{array}{l}3 \cdot 72 \\
3 \cdot 91\end{array}$ & $\begin{array}{l}5 \cdot 81 \\
6 \cdot 64\end{array}$ & $\begin{array}{l}4 \cdot 63 \\
4 \cdot 99\end{array}$ \\
\hline & & $M$ and $F$ & 80 & $5 \cdot 12$ & $4 \cdot 97$ & $3 \cdot 62$ & $3 \cdot 79$ & $6 \cdot 20$ & $4 \cdot 80$ \\
\hline \multirow[t]{2}{*}{${ }^{8}$ and over } & \multirow[t]{2}{*}{$\begin{array}{c}3,629 \\
\text { and over }\end{array}$} & $\underset{\mathrm{F}}{\mathrm{M}}$ & $\begin{array}{l}44 \\
27 \\
\end{array}$ & $\begin{array}{l}5 \cdot 64 \\
6 \cdot 31 \\
\end{array}$ & $\begin{array}{l}5 \cdot 25 \\
5 \cdot 99 \\
\end{array}$ & $\begin{array}{l}3 \cdot 99 \\
4 \cdot 18 \\
\end{array}$ & $\begin{array}{l}4 \cdot 26 \\
4 \cdot 34 \\
\end{array}$ & $\begin{array}{l}7 \cdot 30 \\
8 \cdot 19 \\
\end{array}$ & $\begin{array}{l}5 \cdot 29 \\
5 \cdot 81\end{array}$ \\
\hline & & $M$ and $F$ & 71 & $5 \cdot 89$ & $5 \cdot 53$ & $4 \cdot 06$ & $4 \cdot 29$ & $7 \cdot 64$ & $5 \cdot 49$ \\
\hline
\end{tabular}




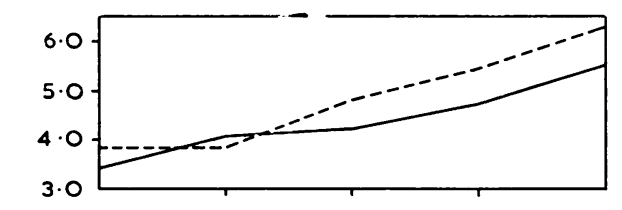

SUBSCAPULAR

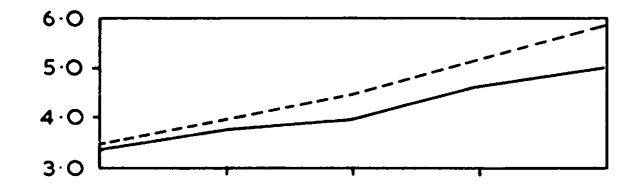

ARM

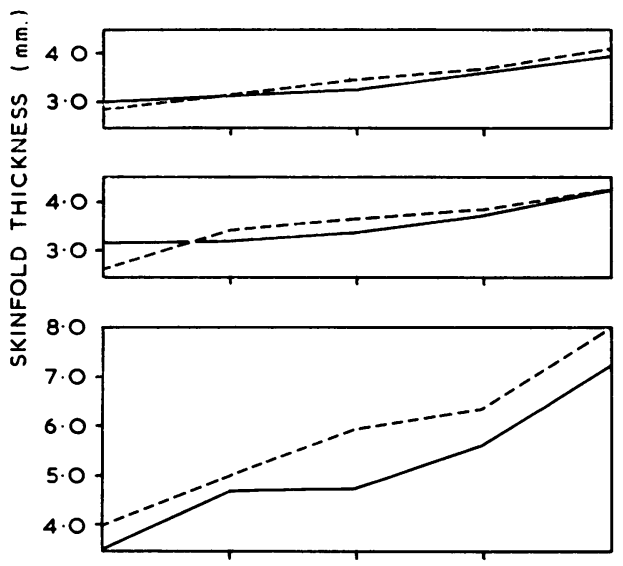

CHEST WALL

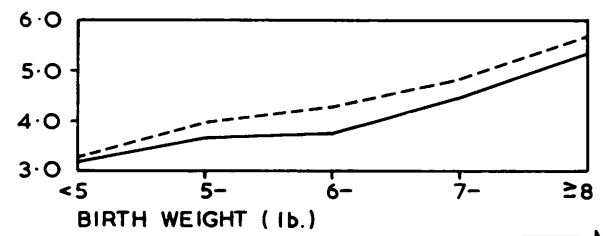

AVERAGE FOR ALL 5 SITES

FIG. 1.-Mean skinfold thickness at each site, and the average for all 5 sites, by weight and sex.

between males and females can be estimated at $0.59 \mathrm{~mm}$. In the 6 sets of unlike twins, taking the average values for all 5 sites, and allowing for weight differences, the mean value for females is $3 \cdot 72 \mathrm{~mm}$. and for males, $3.53 \mathrm{~mm}$. Twins of the same sex and similar weights do not necessarily have similar average skinfold thickness (Table VII). When birth weights differ, the heavier twin may not have the greater average skinfold thickness. The degree of correlation between skinfold thickness and birth weight varies with the site; the correlation coefficient (r) equals approximately 0.4 for the arm, chest wall, and abdomen, 0.5 for the scapula and the average for all the 5 sites, and 0.6 for the thigh (Table IV).

When skinfold thickness is related to the length of gestation (Table V and Fig. 2) the difference between the sexes is still present but is not so marked, and the increase in mean skinfold thickness for each week of gestation is not so constant as it is with weight, tending to be less between 38 and 40 weeks. There is an over-all gain of 0.1 to $0.2 \mathrm{~mm}$. for each week of gestational age depending on the site chosen, being lowest over the chest and abdominal walls and greatest over the thigh. The correlation coefficients are low, varying from 0.2 for the scapula, arm, chest, and abdomen to 0.3 for the thigh (Table VI).

Since birth weight correlates with gestational age and with skinfold thickness, the association between skinfold thickness and length of gestation might have been the result of increasing weight rather than of increasing maturity. The results were therefore 
TABLE IV

Correlation Coefficient of Skinfold Thickness With Birth Weight for 5 Sites

\begin{tabular}{c|c|c|c|c|c|c|}
\hline Scapula & Arm & Chest & Abdomen \\
\hline+0.50 & +0.39 & +0.45 & $+0.42+0.65$ \\
\hline
\end{tabular}

TABLE V

Mean Values of Skinfold Thickness at 5 Sites, by Gestational Age and Sex

\begin{tabular}{|c|c|c|c|c|c|c|c|c|}
\hline $\begin{array}{c}\text { Gestation } \\
\text { (wk.) }\end{array}$ & Sex & No. & $\underset{\text { (mm.) }}{\text { Scapula }}$ & $\underset{(\mathrm{mm} .)}{\operatorname{Arm}}$ & $\begin{array}{l}\text { Chest } \\
\text { (mm.) }\end{array}$ & $\begin{array}{l}\text { Abdomen } \\
\text { (mm.) }\end{array}$ & $\begin{array}{l}\text { Thigh } \\
\text { (mm.) }\end{array}$ & $\begin{array}{c}\text { Average } \\
\text { (mm.) }\end{array}$ \\
\hline \multirow[t]{2}{*}{ Under 37} & $\underset{\mathbf{F}}{\mathbf{M}}$ & $\begin{array}{l}29 \\
13\end{array}$ & $\begin{array}{l}4 \cdot 31 \\
4 \cdot 30\end{array}$ & $\begin{array}{l}4 \cdot 08 \\
3 \cdot 99\end{array}$ & $\begin{array}{l}3 \cdot 30 \\
3 \cdot 31\end{array}$ & $\begin{array}{l}3 \cdot 26 \\
3 \cdot 11\end{array}$ & $\begin{array}{l}4 \cdot 79 \\
5 \cdot 04\end{array}$ & $\begin{array}{l}3.93 \\
3.97\end{array}$ \\
\hline & $M$ and $F$ & 42 & $4 \cdot 31$ & $4 \cdot 05$ & $3 \cdot 30$ & $3 \cdot 21$ & $4 \cdot 89$ & $3 \cdot 95$ \\
\hline \multirow[t]{2}{*}{ 37- } & $\underset{\mathrm{F}}{\mathrm{M}}$ & $\begin{array}{l}17 \\
14\end{array}$ & $\begin{array}{l}4 \cdot 05 \\
4 \cdot 88\end{array}$ & $\begin{array}{l}3 \cdot 98 \\
4 \cdot 82\end{array}$ & $\begin{array}{l}3 \cdot 07 \\
3 \cdot 56\end{array}$ & $\begin{array}{l}3 \cdot 31 \\
3 \cdot 82\end{array}$ & $\begin{array}{l}4 \cdot 69 \\
6 \cdot 61\end{array}$ & $\begin{array}{l}3 \cdot 83 \\
4 \cdot 74\end{array}$ \\
\hline & $M$ and $F$ & 31 & $4 \cdot 43$ & $4 \cdot 36$ & $3 \cdot 29$ & $3 \cdot 54$ & $5 \cdot 56$ & $4 \cdot 24$ \\
\hline \multirow[t]{2}{*}{$38-$} & $\underset{F}{M}$ & $\begin{array}{l}21 \\
26\end{array}$ & $\begin{array}{l}4 \cdot 92 \\
4 \cdot 95\end{array}$ & $\begin{array}{l}4 \cdot 63 \\
4 \cdot 85\end{array}$ & $\begin{array}{l}3 \cdot 61 \\
3 \cdot 54\end{array}$ & $\begin{array}{l}3 \cdot 90 \\
3 \cdot 90\end{array}$ & $\begin{array}{l}5 \cdot 73 \\
6 \cdot 18\end{array}$ & $\begin{array}{l}4 \cdot 52 \\
4 \cdot 70\end{array}$ \\
\hline & $M$ and $F$ & 47 & $4 \cdot 94$ & $4 \cdot 75$ & $3 \cdot 58$ & $3 \cdot 90$ & $5 \cdot 98$ & $4 \cdot 62$ \\
\hline \multirow[t]{2}{*}{ 39- } & $\begin{array}{c}\mathbf{M} \\
\mathbf{F}\end{array}$ & $\begin{array}{l}36 \\
39\end{array}$ & $\begin{array}{l}4 \cdot 78 \\
5 \cdot 16\end{array}$ & $\begin{array}{l}4 \cdot 53 \\
4 \cdot 92\end{array}$ & $\begin{array}{l}3 \cdot 46 \\
3 \cdot 47\end{array}$ & $\begin{array}{l}3 \cdot 60 \\
3 \cdot 60\end{array}$ & $\begin{array}{l}5 \cdot 75 \\
6 \cdot 14\end{array}$ & $\begin{array}{l}4 \cdot 49 \\
4 \cdot 65\end{array}$ \\
\hline & $M$ and $F$ & 75 & $4 \cdot 99$ & $4 \cdot 69$ & $3 \cdot 47$ & $3 \cdot 60$ & $5 \cdot 96$ & $4 \cdot 57$ \\
\hline \multirow[t]{2}{*}{$40-$} & $\underset{\mathrm{F}}{\mathrm{M}}$ & $\begin{array}{l}34 \\
25 \\
\end{array}$ & $\begin{array}{l}4 \cdot 61 \\
5 \cdot 02 \\
\end{array}$ & $\begin{array}{l}4 \cdot 45 \\
4 \cdot 98 \\
\end{array}$ & $\begin{array}{l}3 \cdot 50 \\
3 \cdot 53 \\
\end{array}$ & $\begin{array}{l}3 \cdot 62 \\
3 \cdot 85 \\
\end{array}$ & $\begin{array}{l}6 \cdot 30 \\
6 \cdot 51 \\
\end{array}$ & $\begin{array}{l}4 \cdot 49 \\
4 \cdot 78 \\
\end{array}$ \\
\hline & $M$ and $F$ & 59 & $4 \cdot 78$ & $4 \cdot 67$ & $3 \cdot 51$ & $3 \cdot 72$ & $6 \cdot 39$ & $4 \cdot 61$ \\
\hline \multirow[t]{2}{*}{40 and over } & $\underset{\mathrm{F}}{\mathrm{M}}$ & $\begin{array}{l}25 \\
21 \\
\end{array}$ & $\begin{array}{l}5 \cdot 30 \\
5 \cdot 44 \\
\end{array}$ & $\begin{array}{l}5 \cdot 28 \\
5 \cdot 05 \\
\end{array}$ & $\begin{array}{l}3.93 \\
3.96 \\
\end{array}$ & $\begin{array}{l}4 \cdot 00 \\
3 \cdot 79 \\
\end{array}$ & $\begin{array}{l}6 \cdot 51 \\
6 \cdot 62 \\
\end{array}$ & $\begin{array}{l}5 \cdot 00 \\
4 \cdot 92 \\
\end{array}$ \\
\hline & $M$ and $F$ & 46 & $5 \cdot 36$ & $5 \cdot 17$ & $3 \cdot 94$ & $3 \cdot 91$ & $6 \cdot 56$ & $4 \cdot 97$ \\
\hline
\end{tabular}

TABLE VI

Correlation Coefficient of Skinfold Thickness With Gestational Age for 5 Sites

\begin{tabular}{|c|c|c|c|c|c|}
\hline Scapula & Arm & Chest & Abdomen & Thigh & Average of 5 Sites \\
\hline$+0 \cdot 21$ & $+0 \cdot 20$ & $+0 \cdot 18$ & $+0 \cdot 18$ & $+0 \cdot 30$ & +0.21 \\
\hline
\end{tabular}

divided into 3 weight groups, i.e. $<5 \mathrm{lb} .(2,268 \mathrm{~g}$.), $5 \mathrm{lb}$. to $6 \mathrm{lb}$. $15 \frac{3}{4} \mathrm{oz}$. (3,174 g.), and $7 \mathrm{lb}$. (3,175 g.) and over; and into 3 gestational age-groups, i.e. 36 weeks 6 days and under, 37 weeks to 39 weeks 6 days, and 40 weeks and over. The changes in skinfold thickness with increasing length of gestation differed slightly in each weight group, though there was a tendency for the mean values to fall with increasing maturity (Table VIII and Fig. 3). This trend was most evident in the lightest group at all sites.

The tendency to lower values for skinfold thickness with increasing age is not so pronounced in the intermediate weight group, and in the heavier babies there is an increase in skinfold thickness with increasing maturity at certain sites, viz. over the thigh, arm, and abdomen. On the average, however, there is a downward trend even in this group.

\section{Discussion}

The sites chosen for measurement of skinfold thickness and the methods used have varied with different workers, but most have employed either radiography or some type of pinch caliper. Skinfold calipers were preferred to radiological methods in the present series, as being more suitable for the age-group under study. Garn (1956) used both 
TABLE VII

Average Skinfold Thickness for all Five Sites in 11 Sets of Twins

\begin{tabular}{|c|c|c|c|c|}
\hline \multirow{2}{*}{$\begin{array}{c}\text { Gestation } \\
\text { (wk.) }\end{array}$} & \multirow{2}{*}{ Sex } & \multicolumn{2}{|c|}{ Birth Weight } & \multirow{2}{*}{$\begin{array}{c}\text { Average Skinfold } \\
\text { Thickness } \\
(\mathrm{mm} .)^{\star}\end{array}$} \\
\hline & & lb. & g. & \\
\hline 34 & $\underset{\mathrm{F}}{\mathrm{F}}$ & $\begin{array}{lr}6 & 31 \\
4 & 134\end{array}$ & $\begin{array}{l}2,807 \\
2,183\end{array}$ & $\begin{array}{l}3 \cdot 80 \\
5 \cdot 06\end{array}$ \\
\hline 34 & $\begin{array}{l}M \\
M\end{array}$ & $\begin{array}{rr}5 & 0 \frac{3}{4} \\
4 & 15 \frac{1}{2}\end{array}$ & $\begin{array}{l}2,296 \\
2,240\end{array}$ & $\begin{array}{l}4 \cdot 80 \\
4 \cdot 00\end{array}$ \\
\hline 36 & $\begin{array}{l}\mathbf{M} \\
\mathbf{M}\end{array}$ & $\begin{array}{ll}5 & 23 \\
5 & 5\end{array}$ & $\begin{array}{l}2,353 \\
2,410\end{array}$ & $\begin{array}{l}4 \cdot 06 \\
3 \cdot 42\end{array}$ \\
\hline 37 & $\begin{array}{l}M \\
M\end{array}$ & $\begin{array}{lr}5 & 7 \\
5 & 10\end{array}$ & $\begin{array}{l}2,466 \\
2,552\end{array}$ & $\begin{array}{l}4 \cdot 02 \\
3.94\end{array}$ \\
\hline 37 & $\begin{array}{l}M \\
M\end{array}$ & $\begin{array}{lr}5 & 7 \\
4 & 13\end{array}$ & $\begin{array}{l}2,466 \\
2,183\end{array}$ & $\begin{array}{l}3 \cdot 48 \\
3 \cdot 18\end{array}$ \\
\hline 34 & $\underset{M}{\mathrm{~F}}$ & $\begin{array}{ll}4 & 8 \\
4 & 4 \frac{1}{2}\end{array}$ & $\begin{array}{l}2,041 \\
1,928\end{array}$ & $\begin{array}{l}4 \cdot 20 \\
3 \cdot 50(3 \cdot 61)\end{array}$ \\
\hline 37 & $\underset{\mathrm{M}}{\mathrm{F}}$ & $\begin{array}{cc}7 & 0 \\
6 & 14 \frac{1}{2}\end{array}$ & $\begin{array}{l}3,175 \\
3,119\end{array}$ & $\begin{array}{l}4 \cdot 54 \\
4 \cdot 62(4 \cdot 66)\end{array}$ \\
\hline 37 & $\underset{M}{\mathrm{~F}}$ & $\begin{array}{ll}4 & 14 \\
4 & 12 \frac{1}{2}\end{array}$ & $\begin{array}{l}2,211 \\
2,155\end{array}$ & $\begin{array}{l}2 \cdot 94 \\
2 \cdot 80(2 \cdot 84)\end{array}$ \\
\hline 37 & $\underset{M}{F}$ & $\begin{array}{lr}5 & 11 \\
6 & 1\end{array}$ & $\begin{array}{l}2,580 \\
2,750\end{array}$ & $\begin{array}{l}4 \cdot 90 \\
5 \cdot 52(5 \cdot 31)\end{array}$ \\
\hline 39 & $\underset{\mathrm{M}}{\mathrm{F}}$ & $\begin{array}{l}314 \frac{1}{2} \\
610\end{array}$ & $\begin{array}{l}1,758 \\
3,005\end{array}$ & $\begin{array}{l}2 \cdot 20 \\
3 \cdot 20(1 \cdot 69)\end{array}$ \\
\hline 40 & $\underset{M}{F}$ & $\begin{array}{l}5 \quad 8 \frac{1}{2} \\
614\end{array}$ & $\begin{array}{l}2,500 \\
3,119\end{array}$ & $\begin{array}{l}3 \cdot 52 \\
4 \cdot 01(3 \cdot 27)\end{array}$ \\
\hline
\end{tabular}

$\star$ Values in brackets are the estimated values allowing for differences in weight.

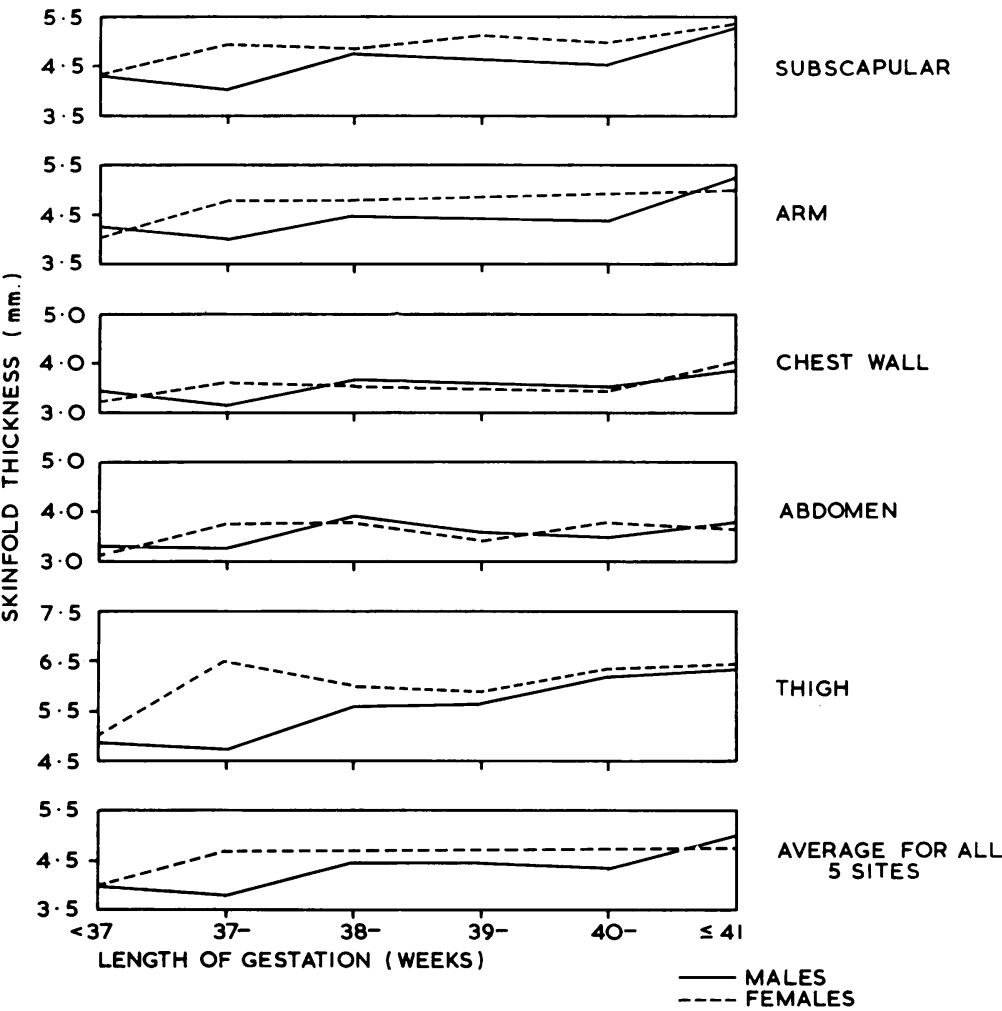

FIG. 2.-Mean skinfold thickness at each site, and the average for all 5 sites, by gestational age and sex. 
TABLE VIII

Mean Skinfold Thickness Values for a Given Weight at Different Periods of Gestation

\begin{tabular}{|c|c|c|c|c|c|c|c|c|c|c|}
\hline \multicolumn{2}{|c|}{ Birth Weight } & \multirow{2}{*}{$\begin{array}{l}\text { Gestation } \\
\text { (wk.) }\end{array}$} & \multirow{2}{*}{ Sex } & \multirow{2}{*}{ No. } & \multirow{2}{*}{$\begin{array}{c}\text { Scapula } \\
\text { (mm.) }\end{array}$} & \multirow{2}{*}{$\underset{(\mathrm{mm} .)}{\operatorname{Arm}}$} & \multirow{2}{*}{$\begin{array}{l}\text { Chest } \\
\text { (mm.) }\end{array}$} & \multirow{2}{*}{$\begin{array}{l}\text { Abdomen } \\
\text { (mm.) }\end{array}$} & \multirow{2}{*}{$\begin{array}{l}\text { Thigh } \\
\text { (mm.) }\end{array}$} & \multirow{2}{*}{$\underset{(\mathrm{mm} .)}{\text { Average }}$} \\
\hline lb. & g. & & & & & & & & & \\
\hline \multirow[t]{5}{*}{$<5$} & \multirow[t]{5}{*}{$<2,268$} & \multirow[t]{2}{*}{$\begin{array}{c}\text { Under } \\
37\end{array}$} & $\underset{\mathbf{F}}{\mathbf{M}}$ & $\begin{array}{r}10 \\
9 \\
\end{array}$ & $\begin{array}{l}3 \cdot 49 \\
4 \cdot 55\end{array}$ & $\begin{array}{l}3 \cdot 49 \\
3 \cdot 94 \\
\end{array}$ & $\begin{array}{l}3 \cdot 12 \\
3 \cdot 27\end{array}$ & $\begin{array}{l}3 \cdot 15 \\
2 \cdot 95\end{array}$ & $\begin{array}{l}3 \cdot 74 \\
4 \cdot 69\end{array}$ & $\begin{array}{l}3 \cdot 40 \\
3 \cdot 82\end{array}$ \\
\hline & & & $\mathbf{M}$ and $\mathbf{F}$ & 19 & $3 \cdot 82$ & $3 \cdot 70$ & $3 \cdot 19$ & $3 \cdot 06$ & $4 \cdot 19$ & $3 \cdot 60$ \\
\hline & & \multirow[t]{2}{*}{$37-$} & $\underset{\mathbf{F}}{\mathbf{M}}$ & $\begin{array}{l}4 \\
7 \\
\end{array}$ & $\begin{array}{l}3 \cdot 32 \\
3 \cdot 58\end{array}$ & $\begin{array}{l}3 \cdot 12 \\
3 \cdot 43\end{array}$ & $\begin{array}{l}2 \cdot 62 \\
2 \cdot 53\end{array}$ & $\begin{array}{l}2 \cdot 85 \\
2 \cdot 50\end{array}$ & $\begin{array}{l}3 \cdot 72 \\
3 \cdot 69\end{array}$ & $\begin{array}{l}3 \cdot 12 \\
3 \cdot 16\end{array}$ \\
\hline & & & $M$ and $F$ & 11 & $3 \cdot 50$ & $3 \cdot 32$ & $2 \cdot 56$ & $2 \cdot 63$ & $3 \cdot 71$ & $3 \cdot 15$ \\
\hline & & $\begin{array}{c}40 \\
\text { and over }\end{array}$ & $\underset{\mathrm{F}}{\mathrm{M}}$ & $\begin{array}{l}0 \\
2 \\
\end{array}$ & $\overline{2 \cdot 80}$ & $\overline{2 \cdot 80}$ & $\overline{2 \cdot 05}$ & $\overline{2 \cdot 05}$ & $\overline{2 \cdot 50}$ & $\overline{2 \cdot 53}$ \\
\hline \multirow[t]{6}{*}{$5-$} & \multirow[t]{6}{*}{$2,268-$} & \multirow[t]{2}{*}{$\underset{37}{\text { Under }}$} & $\begin{array}{c}\mathbf{M} \\
\mathbf{F}\end{array}$ & $\begin{array}{r}15 \\
3 \\
\end{array}$ & $\begin{array}{r}4 \cdot 39 \\
4 \cdot 33 \\
\end{array}$ & $\begin{array}{l}4 \cdot 15 \\
4 \cdot 23 \\
\end{array}$ & $\begin{array}{l}3 \cdot 27 \\
3 \cdot 50 \\
\end{array}$ & $\begin{array}{l}3 \cdot 29 \\
3 \cdot 60\end{array}$ & $\begin{array}{l}5 \cdot 10 \\
5 \cdot 70\end{array}$ & $\begin{array}{l}4 \cdot 04 \\
4 \cdot 27\end{array}$ \\
\hline & & & $M$ and $F$ & 18 & $4 \cdot 38$ & $4 \cdot 17$ & $3 \cdot 32$ & $3 \cdot 34$ & $5 \cdot 20$ & $4 \cdot 08$ \\
\hline & & \multirow[t]{2}{*}{$37-$} & $\underset{\mathrm{F}}{\mathrm{M}}$ & $\begin{array}{l}35 \\
39 \\
\end{array}$ & $\begin{array}{l}4 \cdot 20 \\
4 \cdot 59 \\
\end{array}$ & $\begin{array}{l}4 \cdot 12 \\
4 \cdot 44 \\
\end{array}$ & $\begin{array}{l}3 \cdot 16 \\
3 \cdot 32 \\
\end{array}$ & $\begin{array}{l}3.42 \\
3.63 \\
\end{array}$ & $\begin{array}{l}4 \cdot 95 \\
5 \cdot 72\end{array}$ & $\begin{array}{l}3 \cdot 96 \\
4 \cdot 34\end{array}$ \\
\hline & & & $M$ and $F$ & 74 & $4 \cdot 40$ & $4 \cdot 29$ & $3 \cdot 25$ & $3 \cdot 53$ & $5 \cdot 35$ & $4 \cdot 16$ \\
\hline & & \multirow[t]{2}{*}{$\begin{array}{c}40 \\
\text { and over }\end{array}$} & $\underset{\mathrm{F}}{\mathrm{M}}$ & $\begin{array}{l}12 \\
13 \\
\end{array}$ & $\begin{array}{l}4 \cdot 02 \\
4 \cdot 23 \\
\end{array}$ & $\begin{array}{l}3 \cdot 66 \\
4 \cdot 21 \\
\end{array}$ & $\begin{array}{l}3 \cdot 11 \\
3 \cdot 29 \\
\end{array}$ & $\begin{array}{l}3 \cdot 10 \\
3 \cdot 41 \\
\end{array}$ & $\begin{array}{l}4 \cdot 63 \\
5 \cdot 69 \\
\end{array}$ & $\begin{array}{l}3 \cdot 70 \\
4 \cdot 17 \\
\end{array}$ \\
\hline & & & $M$ and $F$ & 25 & $4 \cdot 13$ & $3 \cdot 95$ & $3 \cdot 20$ & $3 \cdot 26$ & $5 \cdot 18$ & $3 \cdot 94$ \\
\hline \multirow[t]{6}{*}{ and over } & \multirow[t]{6}{*}{$\begin{array}{c}3,175 \\
\text { and over }\end{array}$} & \multirow[t]{2}{*}{$\underset{37}{\text { Under }}$} & $\underset{\mathrm{F}}{\mathrm{M}}$ & $\begin{array}{l}4 \\
1 \\
\end{array}$ & $\begin{array}{l}6 \cdot 08 \\
4 \cdot 70\end{array}$ & $\begin{array}{l}5 \cdot 30 \\
3 \cdot 70\end{array}$ & $\begin{array}{l}3 \cdot 82 \\
3 \cdot 10\end{array}$ & $\begin{array}{l}3 \cdot 40 \\
3 \cdot 10\end{array}$ & $\begin{array}{l}6 \cdot 22 \\
6 \cdot 20\end{array}$ & $\begin{array}{l}5 \cdot 48 \\
4 \cdot 30\end{array}$ \\
\hline & & & $M$ and $F$ & 5 & $5 \cdot 80$ & $4 \cdot 98$ & $3 \cdot 68$ & $3 \cdot 34$ & $6 \cdot 22$ & $5 \cdot 22$ \\
\hline & & \multirow[t]{2}{*}{$37-$} & $\underset{\mathrm{F}}{\mathrm{M}}$ & $\begin{array}{l}35 \\
33 \\
\end{array}$ & $\begin{array}{l}5 \cdot 28 \\
5 \cdot 88 \\
\end{array}$ & $\begin{array}{l}4 \cdot 81 \\
5 \cdot 69 \\
\end{array}$ & $\begin{array}{l}3 \cdot 75 \\
3 \cdot 94 \\
\end{array}$ & $\begin{array}{l}3 \cdot 90 \\
4 \cdot 13 \\
\end{array}$ & $\begin{array}{l}6 \cdot 26 \\
7 \cdot 41 \\
\end{array}$ & $\begin{array}{l}4 \cdot 80 \\
5 \cdot 41 \\
\end{array}$ \\
\hline & & & $M$ and $F$ & 68 & $5 \cdot 57$ & $5 \cdot 24$ & $3 \cdot 84$ & $4 \cdot 01$ & $6 \cdot 82$ & $5 \cdot 10$ \\
\hline & & \multirow[t]{2}{*}{$\begin{array}{c}40 \\
\text { and over }\end{array}$} & $\underset{\mathrm{F}}{\mathrm{M}}$ & $\begin{array}{l}47 \\
31 \\
\end{array}$ & $\begin{array}{l}5 \cdot 13 \\
5 \cdot 77 \\
\end{array}$ & $\begin{array}{l}5 \cdot 09 \\
5 \cdot 49 \\
\end{array}$ & $\begin{array}{l}3 \cdot 83 \\
3 \cdot 83 \\
\end{array}$ & $\begin{array}{l}4 \cdot 04 \\
4 \cdot 02 \\
\end{array}$ & $\begin{array}{l}6 \cdot 83 \\
7 \cdot 18 \\
\end{array}$ & $\begin{array}{l}4 \cdot 97 \\
5 \cdot 29\end{array}$ \\
\hline & & & $\mathbf{M}$ and $\mathbf{F}$ & 78 & $5 \cdot 38$ & $5 \cdot 28$ & $3 \cdot 83$ & $4 \cdot 03$ & $6 \cdot 97$ & $5 \cdot 10$ \\
\hline
\end{tabular}

radiographs and calipers, and found a high correlation between the results when measuring skinfold thickness at the level of the lowest ribs in the midaxillary line. Garn (1958), Singh and Venkatachalam (1962), and Stuart and Sobel (1946) measured skinfold thickness over the lower leg, and Standard, Wills, and Waterlow (1959), Singh and Venkatachalam (1962), and Vincent and Hugon (1962) measured it over the triceps. Standard et al. also took readings from the lateral, medial, and anterior aspects of the thigh. Several sites were used in the present study because it seemed possible that measurements at different sites might show different changes in skinfold thickness, and that one site might be of more value than the others.

It might be expected that skinfold thickness would have a high correlation with birth weight which itself is related to gestational age. The coefficient of correlation between weight and gestation in this study was 0.4 , which is in agreement with values of $0 \cdot 3-0 \cdot 5$ for other series (Karn and Penrose, 1951). Garn (1958) measured skinfold thickness in
146 infants and found that fat thickness was moderately related to birth weight in both sexes $(r=0.6$ to $0 \cdot 7$ ). Since body weight does not differentiate between the amount of protoplasmic tissue, fat, water, storage, bone, and muscle (Stuart and Meredith, 1946), there may be differences in skinfold thickness for infants of the same weight but of different gestational age. Vincent and Hugon (1962) found that measurements over the arm and back gave similar values until term when those over the scapula became greater than those over the triceps.

The present results show that there is an increase in skinfold thickness with increasing birth weight. The increase in thickness over the thigh is the most marked, and follows most nearly the pattern found for the average of the five sites. The mean values over the arm and scapula are smaller than those obtained by Singh and Venkatachalam (1962); this may be a real difference, as the crown-heel lengths of the infants are similar in the two series, but it could be the result of using different types of caliper. 


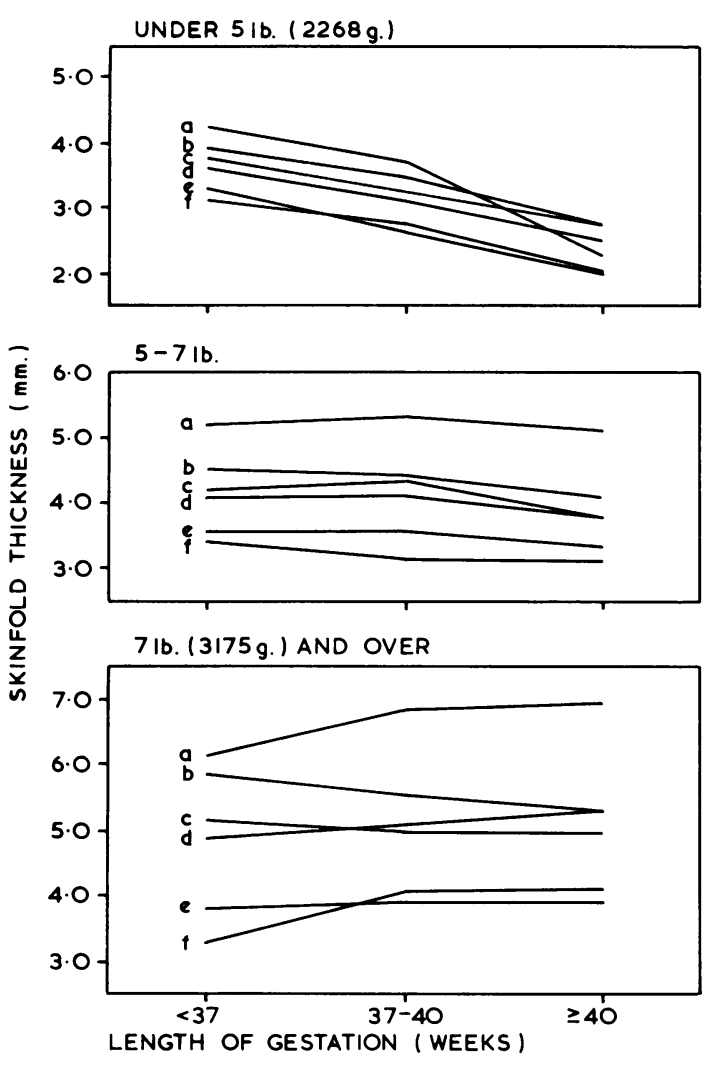

FIG. 3.-Mean skinfold thickness for a given weight at different periods of gestation. $a$, thigh; $b$, scapula; $c$, arm; $d$, average of the five sites; $e$, chest wall; $f$, abdomen.

The higher mean values for females agree with the results of Vincent and Hugon (1962) and Gampel (1965), and may be partly related to the fact that females are shorter than males of the same weight, particularly in the heavier groups. The tendency to diminishing skinfold thickness with increasing maturity in infants of the same birth weight may also be partly explained by the increase in body length as gestational age increases, and Gampel (1965) shows that post-term infants increase in size but do not put on a proportionate amount of subcutaneous tissue.

Measurement of skinfold thickness is of no value as an indication of maturity. Estimation of gestational age based on skinfold thickness is subject to a standard deviation of $2 \cdot 2$ weeks for males and $2 \cdot 0$ weeks for females, and is less accurate than could be made by taking birth weight alone. The positive association between length of gestation and skinfold thickness appears to result from the association of birth weight and gestation on the one hand, and of birth weight and skinfold thickness on the other. The correlation between gestation and skinfold thickness becomes negligible when the effect of birth weight is eliminated.

\section{Summary}

Skinfold thickness was measured in 162 males and 138 females under 48 hours of age and of varied gestational ages, and the results were related to birth weight and gestational age. Five sites were chosen - scapula, arm, chest wall, abdominal wall, and anterior thigh. Mean values for skinfold thickness were higher in females than in males; given birth weight and maturity, this difference can be estimated as $0.59 \mathrm{~mm}$. In the whole series of 300 infants, taking the average of the 5 sites chosen, there was a correlation with weight of 0.6 ; with length of gestation, the correlation was $0 \cdot 2$ and was shown to result from the correlations between birth weight and gestational age and birth weight and skinfold thickness. When both weight and gestational age were taken into account, there was a tendency for skinfold thickness to fall with increasing maturity in infants of a given weight. It is concluded that measurement of skinfold thickness is of no value in estimating the length of gestation of a newborn infant.

I wish to thank Professor R. G. Mitchell for his help and encouragement, Mr. W. Z. Billewicz, M.R.C. Statistical Officer, for statistical advice, and Mr. E. Rae, the Department of Materia Medica and Therapeutics, for technical assistance.

\section{REFERENCES}

Gampel, B. (1965). The relation of skinfold thickness in the neonate to sex, length of gestation, size at birth and maternal skinfold. Hum. Biol., 37, 29.

Garn, S. M. (1956). Comparison on pinch-caliper and X-ray measurements of skin plus subcutaneous fat. Science, 124, 178 . (1958). Fat, body size and growth in the newborn. Hum. Biol., 30, 265.

Karn, M. N., and Penrose, L. S. (1951). Birth weight and gestation time in relation to maternal age, parity and infant survival. Ann. Eugen. (Lond.), 16, 147.

Parmelee, A. H. (1959). Characteristics of the newborn. In Management of the Newborn, 2nd ed., chap. 4. Year Book Publishers, Chicago.

Singh, R., and Venkatachalam, P. S. (1962). Anthropometric studies of the new born. Indian f. med. Res., 50, 794.

Standard, K. L., Wills, V. G., and Waterlow, J. C. (1959). Indirect indicators of muscle mass in malnourished children. Amer. F. clin. Nutr., 7, 271.

Stuart, H. C., and Meredith, H. V. (1946). Use of body measurements in the school health program. Amer. F. publ. Hlth, 36, 1365.

- - and Sobel, E. H. (1946). The thickness of the skin and subcutaneous tissue, by age and sex, in childhood. F. Pediat., $28,637$.

Verel, D., and Kesterven, A. B. (1960). Forceps for the measurement of skin-fold thickness. Lancet, 2, 962.

Vincent, M., and Hugon, J. (1962). L'insuffisance pondérale du prématuré africain. Bull. Wld Hlth Org., 26, 143. 


\section{Appendix}

\section{Comparison of Calipers for Measurement of Skinfold Thickness in Neonatal Period}

Most of the calipers at present available for the measurement of skinfold thickness are too large and unwieldy for use in infants, particularly in the neonatal period. Verel and Kesterven (1960) described a small set of calipers suitable for children; these have been compared with the widely used Harpenden calipers, both for ease of handling and for comparability of results (see Table).

Procedure. Measurements were made in 65 infants, their ages ranging from 4 hours to 24 days. Both premature and full-term babies were included so that a wide range of values for skinfold thickness could be compared. Measurements were made over the lower rib margin, in the nipple line, and over the lower angle of the scapula, both on the left side. One reading was made with each set of calipers at each site. The first 60 readings were made in 30 infants with a mean age of 3.5 days, comparing the Harpenden calipers with the original Verel and Kesterven calipers which have a scale in $2.5 \mathrm{~mm}$. divisions. For the second group of 35 infants with a mean age of 8.0 days, the Verel scale was modified by making the divisions of $1.25 \mathrm{~mm}$. and using a perspex of greater magnification. This modified instrument can be read to $0.6 \mathrm{~mm}$. by interpolation between the divisions.

\section{TABLE}

Number of Cases in Each Group When Values Obtained With Verel and Kesterven Calipers are Subtracted from Those Obtained With Harpenden Calipers

\begin{tabular}{|c|c|c|c|c|}
\hline \multirow{2}{*}{$\begin{array}{l}\text { Deviation } \\
\text { (mm.) }\end{array}$} & \multicolumn{2}{|c|}{$\begin{array}{l}\text { Using } 2.5 \mathrm{~mm} \text {. Scale } \\
(30 \text { cases })\end{array}$} & \multicolumn{2}{|c|}{ Using $\underset{\text { (35 cases) }}{1 \cdot 25 \mathrm{~mm}}$. Scale } \\
\hline & $\underset{\text { lar }}{\text { Subscapu- }}$ & $\begin{array}{l}\text { Chest } \\
\text { Wall }\end{array}$ & $\begin{array}{c}\text { Subscapu- } \\
\text { lar }\end{array}$ & $\begin{array}{l}\text { Chest } \\
\text { Wall }\end{array}$ \\
\hline $\begin{array}{l}+1.0 \\
+0.9 \\
+0.8 \\
+0.7 \\
+0.6 \\
+0.5 \\
+0.4 \\
+0.3 \\
+0.2 \\
+0.1 \\
0.0 \\
-0.1 \\
-0.2 \\
-0.3 \\
-0.4 \\
-0.5 \\
-0.6 \\
-0.7\end{array}$ & $\begin{array}{l}\overline{7} \\
\frac{1}{1} \\
\frac{1}{10} \\
10 \\
2 \\
2 \\
2 \\
1 \\
1 \\
\frac{4}{2} \\
1 \\
2 \\
-\end{array}$ & $\begin{array}{l}3 \\
1 \\
2 \\
1 \\
- \\
2 \\
2 \\
3 \\
3 \\
2 \\
2 \\
2 \\
2 \\
2 \\
2 \\
1\end{array}$ & $\begin{array}{l}\text { 二 } \\
\overline{1} \\
\frac{1}{2} \\
5 \\
6 \\
5 \\
8 \\
3 \\
2 \\
1 \\
2 \\
= \\
=\end{array}$ & $\begin{array}{l}\text { 二 } \\
\overline{2} \\
2 \\
\frac{5}{5} \\
2 \\
7 \\
7 \\
2 \\
3 \\
3 \\
2 \\
= \\
=\end{array}$ \\
\hline
\end{tabular}

Results. In the first 30 infants, the mean values for the subscapular region were $4.0 \mathrm{~mm}$. and $4.1 \mathrm{~mm}$.; those for the anterior chest wall were $2.7 \mathrm{~mm}$. and $2.9 \mathrm{~mm}$. In each case the higher value was obtained with the Harpenden calipers. In 12 instances $(20 \%)$ the difference between the readings was greater than $0.5 \mathrm{~mm}$.; 8 of these readings were taken over the chest wall. Since values for skinfold thickness in this age-group are so low, it was considered that such a difference was too great, and so the scale markings on the Verel and Kesterven calipers were increased. Using the modified calipers, the mean values for the second group of infants were $3.3 \mathrm{~mm}$. and $3.5 \mathrm{~mm}$. for the scapula, and $2.3 \mathrm{~mm}$. and $2.5 \mathrm{~mm}$. for the chest; again, the higher readings were obtained with the Harpenden calipers. Of the 70 recordings, only $5(7 \%)$ showed a difference of more than $0.5 \mathrm{~mm}$.; 4 of these were taken over the anterior chest wall. In all 5 , readings were higher with the Harpenden calipers; indeed, the Harpenden calipers gave higher readings in all but 13 of the 70 recordings $(18.5 \%)$.

Discussion. The difference in the results obtained with the two sets of calipers may have been due to differences in pressure or to differences in the recording mechanism. Both calipers are designed to exert a pressure of $10 \mathrm{~g} . / \mathrm{sq}$. mm., but the Harpenden calipers have a dial type of scale with a spring-loaded indicator which measures to $0.1 \mathrm{~mm}$., whereas the Verel and Kesterven calipers have a fixed scale with an indicator which does not swing. Waiting until the Harpenden indicator had almost come to rest generally caused sufficient discomfort to make the baby wriggle, making accurate reading difficult, and this could have contributed to the higher readings. The Verel calipers can be read very quickly and produce much less disturbance. The scale is not fine enough for the thinner infants, but a slight modification to permit recording to $0.6 \mathrm{~mm}$. results in an improvement in the correlation of results.

Summary. Skinfold thickness was measured in 65 newborn infants, using both the Harpenden and Verel and Kesterven calipers, recordings being made over the lower angle of the scapula and the anterior chest wall. Higher mean values were obtained with the Harpenden calipers, but the percentage of recordings differing by more than $0.5 \mathrm{~mm}$. was reduced from 20 to $7 \%$ when the Verel and Kesterven scale was altered to read to $0.6 \mathrm{~mm}$. The modified Verel and Kesterven calipers are more suitable for use in the newborn since they cause less discomfort and are easier to work with than the Harpenden calipers, advantages that more than offset the greater discrimination of the Harpenden scale. 